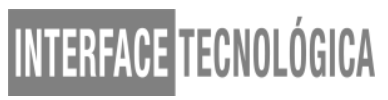

\title{
A IMPORTÂNCIA DO CICLO PDCA APLICADO À PRODUTIVIDADE DA INDÚSTRIA NO BRASIL
}

\author{
THE IMPORTANCE OF THE PDCA CYCLE APPLIED TO INDUSTRY \\ PRODUCTIVITY IN BRAZIL
}

\author{
Valdeci Gomes Filho - leidegomesspo@gmail.com \\ Angelita Moutin Segória Gasparotto - angelita.gasparotto@ fatectq.com.br \\ Faculdade de Tecnologia de Taquaritinga (FATEC) -SP -Brasil \\ DOI: 10.31510/infa.v16i2.660
}

\section{RESUMO}

O Clico PDCA tem sido usado atualmente pelas empresas na execução e seu planejamento estratégico de forma eficiente e dinâmica. A qualidade dos serviços oferecidos pelas empresas é um diferencial necessário para a sua sobrevivência no mercado, sendo assim, a determinação de processos se mostra como fator determinante no desenvolvimento dos negócios, já que o Brasil conta com um contingente de milhares de indústrias, as quais têm grande importância no cenário econômico do país, e uma participação significativa no Produto Interno Bruto (PIB). A utilização do ciclo PDCA no ambiente organizacional é um caminho para melhorar os resultados e alavancar o desempenho das empresas. O Ciclo de Qualidade, como uma metodologia que visa auxiliar no diagnóstico e no prognóstico de problemas nas organizações e possui como foco na melhoria contínua de processos de qualquer natureza. Para sua implementação, é necessário um estudo de todas as suas etapas.

Palavras-chave: Clico PDCA, Desempenho Empresarial, Planejamento Estratégico.

\begin{abstract}
The PDCA Cycle has been used today by companies to execute and their strategic planning efficiently and dynamically. The quality of the services offered by the companies is a necessary differential for their survival in the market. Thus, the determination of processes is a determining factor in business development, since Brazil has a contingent of thousands of industries, which have great importance in the economic scenario of the country, and a significant participation in the Gross Domestic Product (GDP). Using the PDCA cycle in the organizational environment is a way to improve results and leverage business performance. For its implementation, a study of all its steps is required. The Quality Cycle, as a methodology that aims to assist in the diagnosis and prognosis of problems in organizations and focuses on continuous improvement of processes of any kind. For its implementation, a study of all its steps is required.
\end{abstract}

Keywords: PDCA cycle. Business performance. Strategic planning. 


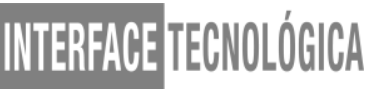

\section{INTRODUÇÃO}

O Ciclo PDCA (Plan-Do-Check-Action) tem sido usado atualmente pelas empresas na execução e seu planejamento estratégico de forma eficiente e dinâmica. A qualidade dos serviços oferecidos pelas empresas é um diferencial necessário para sua sobrevivência no mercado, sendo assim, a determinação de processos se mostra como fator determinante no desenvolvimento dos negócios, já que o Brasil conta com um contingente de milhares de indústrias, as quais têm grande importância no cenário econômico do país, e uma participação significativa no Produto Interno Bruto (PIB) (ABIA, 2016).

A utilização do ciclo PDCA no ambiente organizacional é um caminho para melhorar os resultados e alavancar o desempenho das empresas. De acordo com Fonseca e Miyake (2006), o ciclo PDCA tem foco na melhoria contínua de processos de qualquer natureza. Para sua implementação, é necessário um estudo de todas as suas etapas. Quinquiolo (2002) define o ciclo PDCA, também conhecido como o Ciclo de Qualidade, como uma metodologia que visa auxiliar no diagnóstico e no prognóstico de problemas nas organizações.

$\mathrm{O}$ processo de melhoria contínua tem como base principal as atividades de ruptura e controle e é baseado em uma abordagem científica cujo processo de resolução de problemas pode ser dividido em: a) identificação de causa, b) planejamento e c) padronização da solução (SHIBA; GRAHAM; WALDEN, 1997). A melhoria contínua pode ser traduzida em um ciclo, que se concentra na orientação do planejamento, das ações, controle e melhorias aplicadas.

Segundo Quinquiolo (2002), como justificativas para a utilização do ciclo PDCA, pode-se destacar a produtividade, que está ligada à competividade e ao uso de TICs (Tecnologias de Informação e Comunicação). A gestão busca melhorar os processos com ferramentas da qualidade, ajudando na melhoria contínua dos processos. Uma vez que uma empresa tem seus processos otimizados, o próximo passo é investir em novas tecnologias.

De acordo com Quinquiolo (2002), a competitividade é a base do sucesso ou do fracasso de um negócio onde há livre concorrência. Empresas com boa competitividade prosperam e destacam-se de seus concorrentes. A competitividade pode ser colocada como o ponto principal das grandes organizações empresariais, mas, se usada como uma ferramenta lucrativa, pode se tornar prejudicial às atividades da empresa.

Nesse contexto, o objetivo deste trabalho é evidenciar a significância do ciclo PDCA na aplicação de uma indústria do ramo alimentício por meio dos fatores de produção 


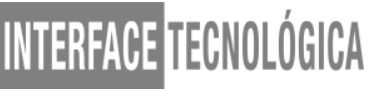

utilizados. Espera-se que a implementação do Ciclo PDCA possa trazer ganhos significativos para a empresa em termos de produtividade e eficiência (SHIBA; GRAHAM; WALDEN, 1997).

\section{CICLO PDCA}

De acordo com Arruda (1997), na década de 20, foi criada a ferramenta de qualidade PDCA, conhecida também como ciclo de Shewhart ou ciclo de Deming. Seu idealizador foi Walter Shewhat e ela foi disseminada mais tarde pelo norte-americano William Edward Deming.

Segundo Arruda (1997), o PDCA é um ciclo que, ao ser implementado constantemente em um processo de trabalho, possibilita altos níveis de melhorias contínuas de desempenho. Para Shiba (1997), o PDCA é representado por um ciclo eficiente na resolução de problemas, que consiste em melhorias por etapas e repetição do ciclo de melhorias várias vezes. Marshall Jr. et al (2008), por sua vez, consideram o PDCA é uma ferramenta gerencial para promoção da melhoria contínua e reflete, em quatro fases, a base da filosofia do melhoramento contínuo.

Para Arruda (1997), a filosofia e as ideias do PDCA são aplicáveis em todas as organizações de qualquer dimensão, podendo ser em produtos, serviços ou até mesmo departamentos ou unidades de uma organização. Assim, o ciclo, quando repetido várias vezes, traz vantagens competitivas ao negócio, fazendo que a qualidade e excelência sejam pontos estratégicos dentro da organização.

Para a execução completa do ciclo PDCA, as seguintes fases são necessárias:

$1^{\circ}$ Fase - Plan (Planejar): esse é a primeira etapa do ciclo de melhoria contínua e é o responsável pelo desenho do processo em que se quer ter o conceito aplicado. Nessa fase é importante ressaltar três sub-etapas: estabelecer objetivos, estabelecer o caminho a partir do qual esse objetivo será alcançado e o método a ser usado (ARRUDA, 1997).

$2^{\circ}$ Fase - Do (Executar): essa fase é a da execução da etapa anterior de planejamento e consiste em treinamentos dos envolvidos, a execução do que foi planejado e coleta de dados para futura análise (ARRUDA, 1997).

$3^{\circ}$ Fase - Check (Verificar ou checar): nessa fase, realizam-se a análise e verificação dos dados coletados anteriormente e também se detectam as possíveis falhas e erros que imprevistos no desenho do processo (ARRUDA, 1997).

Interface Tecnológica - v. 16 n. 2 (2019) 
$4^{\circ}$ Fase - Act (Agir): essa é a última fase do ciclo. Após sua conclusão, o PDCA inicia um novo ciclo. Nesta fase são aprimoradas as correções e definidos os planos de ação para o melhoramento da qualidade, eficiência e eficácia (ARRUDA, 1997).

A ilustração 1, mostra a representação do ciclo PCDA.

\section{Ilustração 1 - Ciclo PDCA}

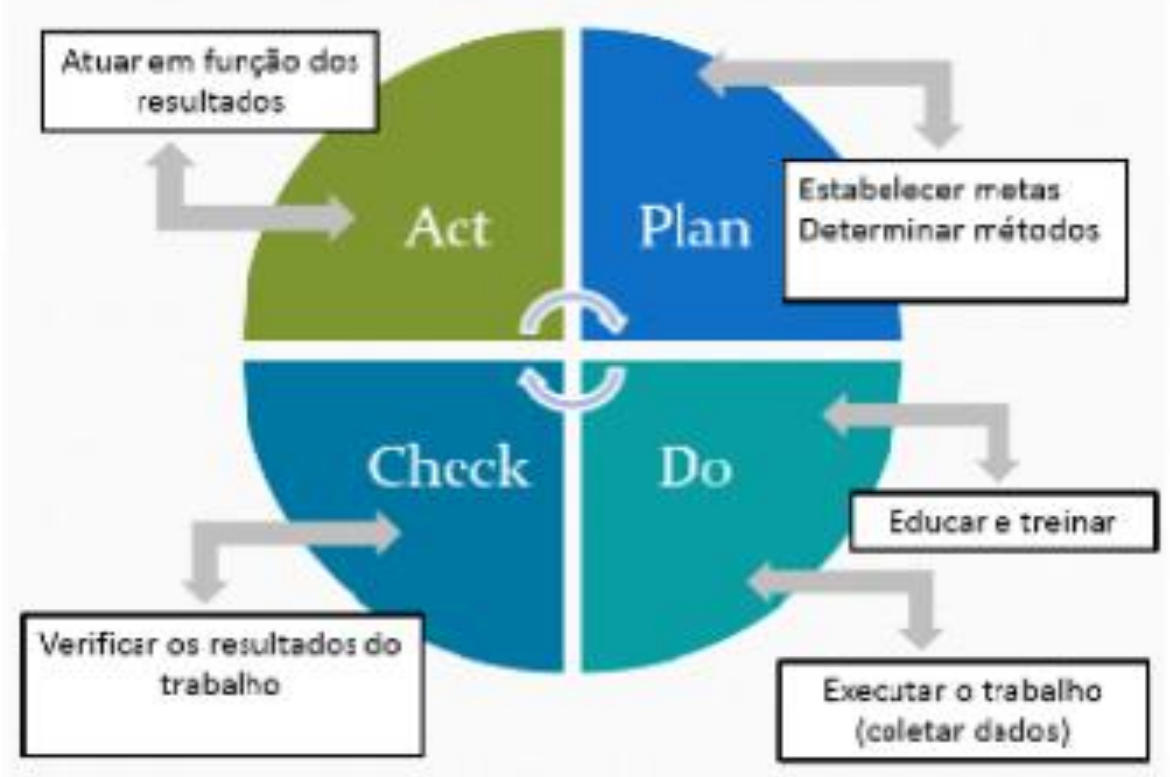

Fonte: Arruda (1997).

A criação do ciclo PDCA permitiu alcançar a previsibilidade nos processos e aumentar a competitividade organizacional. Essa previsibilidade acontece por meio da obediência aos padrões, pois quando a melhoria é bem sucedida, o método planejado é adotado, padronizando-o; caso contrário, ele reverte para o padrão anterior e começa a rodar o PDCA novamente (ARRUDA, 1997). 


\section{|WTERFFAET TECNOLOGGCA}

\section{PROCEDIMENTOS METODOLÓGICOS}

Para a elaboração do artigo foi realizada pesquisa bibliográfica, partindo do levantamento de referências teóricas já analisadas, e publicadas por meios escritos e eletrônicos, como livros, artigos científicos e websites (GIL, 2007).

O estudo em questão é sobre o PDCA aplicado à produtividade da indústria no Brasil, e sua contribuição para o aumento da produtividade, reduzindo as perdas e visando assim a customização do sistema de produção atual para um sistema de produção enxuta (SLACK, 2018).

\section{RESULTADOS E DISCUSSÕES}

A metodologia PDCA é amplamente empregada em processos de manufatura, pois se trata de um instrumento eficiente para análise e para resolução de problemas. Método é um termo que se origina do grego e é composto pelas palavras meta, cujo significado é "além de" todos, que significa "caminho". Assim, a palavra método representa caminho para se chegar a um ponto além do caminho (CAMPOS, 1992).

Adaptando a definição de Campos (1992) ao conceito de PDCA, pode-se concluir que o método é um caminho para se atingir uma meta. Pode-se observar o cuidado no uso dos métodos, tanto de forma qualitativa quanto quantitativa em diversos estudos científicos. Segundo Pereira (2004), os métodos constituem uma ferramenta de se agruparem resultados e obter-se uma melhor compreensão geral acerca de um fenômeno que foi analisado.

Para Coltro (1996) o uso do método PDCA pelas empresas se dá pela eficiência em gerir os processos internos, de maneira a assegurar a aquisição das metas colocadas, utilizando os dados como fonte de direção das decisões.

A fase inicial refere-se ao planejamento (PLAN), no qual se define o escopo do recurso estudado, determinando métodos para seu método. Na segunda etapa, a de execução (DO), é necessário treinamento e envolvimento de pessoas para a execução efetiva do planejamento. A terceira etapa, a de verificação (CHECK), objetiva comparar o que foi obtido com o planejado. Nesta etapa, verifica-se se os resultados foram alcançados. Já a última etapa (ACTION), representa a tomada de ações corretivas, caso aplicável. Se o proposto 


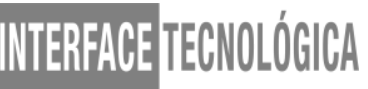

inicialmente foi obtido, deve-se garantir a continuidade do processo, padronizando-o (COLTRO, 1996).

\subsection{Etapa de planejamento (Módulo P - PLAN)}

De acordo com Araújo (2017), a etapa de Planejamento, por ser o início do ciclo PDCA, é considerada a mais significativo, pois nela são estabelecidos os objetivos e os processos indispensáveis para fornecer resultados conforme o solicitado pelo cliente. Portanto, um bom planejamento proverá de todas as informações e dados necessários para a realização completa do procedimento com eficácia.

A etapa do planejamento é sempre a mais complicada e que exige mais esforços, pois, quanto maior for o número de informações utilizadas, maior será a necessidade do emprego de ferramentas para coletar e processar essas informações, além de dispor delas (ARAÚJO, 2017).

Para Araújo (2017), determinadas ferramentas como Diagrama de Ishikawa, Gráfico de Pareto, brainstorming e 5W2H são muito utilizadas nesta fase, para oferecer suporte nas tomadas de decisões. Porque, quanto melhor o planejamento, as metas serão mais fáceis de serem atingidas. Andrade e Melhado (2003) explicam que o módulo planejar abrange várias etapas do processo, classificadas da seguinte forma:

\section{Localizar o problema $\rightarrow$ Estabelecer meta $\rightarrow$ Análise do fenômeno $\rightarrow$ Análise do processo $\rightarrow$ Elaborar plano de ação}

\subsection{Etapa de execução (Módulo D - DO)}

A etapa seguinte é o DO (executar). Para Araújo (2017), nela encontramos todos os objetivos e metas a serem alcançados; traçados em um plano de ação que deve ser muito bem estruturado, e que deverá ser posto em prática na organização escolhida.

Para a melhor eficiência desta fase, Campos (2001) subdivide-a em duas etapas principais: Treinamento e Execução da ação. 


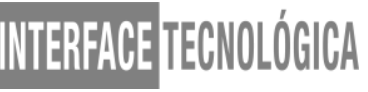

No treinamento, os funcionários envolvidos devem ter conhecimento do plano de ação, divulgado em reuniões, com ênfase nas tarefas e nas suas razões; como também no seu controle. É necessária verificação para identificar qual tarefa necessita da participação de todos os funcionários, buscando a melhor execução possível. Com a intenção de se possuir um controle mais preciso das medidas apresentadas no plano de ação, é necessário fazer a medição regular e adequada desses itens de controle, que poderão estar sendo gerados quando uma nova meta for proposta ou poderão estar locadas na manutenção rotineira (CAMPOS, 2001).

\subsection{Etapa de checagem (Módulo C - CHECK)}

A terceira fase do ciclo PDCA é o Check (verificar), de acordo com Araújo (2017, em que ocorre a verificação do que foi executado na fase anterior (DO). Para que ocorra a verificação da melhor maneira possível, esta fase baseia-se nas ações que vem da etapa de planejamento, sendo assim, todas as ações deverão ser monitoradas e formalizadas na fase executar.

Conforme Melo (2001), para a melhor organização, esta etapa é dividida em três fases: listagens dos efeitos secundários, verificação da continuidade ou não do problema, e comparação dos resultados. Dessa maneira, a fase é organizada para uma melhor eficácia da ação.

Ainda de acordo com o autor, primeiro deve-se fazer a comparação dos resultados, utilizando os dados coletados na fase anterior, com a finalidade de se verificarem o grau de redução dos problemas e o grau de eficácia das ações. Logo após a execução da etapa de comparação dos resultados, podendo provocar efeitos secundários negativos ou positivos para a empresa. A etapa seguinte é a da listagem dos efeitos secundários, em que serão tomadas as melhores decisões sobre esses efeitos. Para finalizar, deve-se fazer a verificação da continuidade ou não do problema, quando o saldo das ações é aceitável em relação ao esperado. A organização, levando em consideração o plano inicial, deve garantir que todas as ações planejadas sejam executadas. Caso os efeitos negativos continuassem a ocorrer mesmo depois do implemento das ações planejadas, a solução apresentada se configuraria como falha. Dessa maneira, para que as causas desses problemas possam ser solucionadas, deve-se 


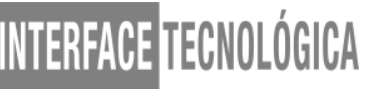

reiniciar o ciclo PDCA. Caso contrário, se os efeitos forem positivos, tendo o êxito das ações tomadas, a empresa poderá passar para a próxima fase o ACTION (ATUAR) do ciclo PDCA.

\subsection{Etapa de ação corretiva (Módulo A - ACTION)}

Esta última etapa do ciclo PDCA, segundo Araújo (2017), tem como base a padronização, que incide em preparar um novo padrão, ou modificar o já existente das ações efetuadas, cuja eficiência foi averiguada na etapa precedente, tendo por objetivo a melhoria contínua.

Para Araújo (2017), logo após a elaboração dos novos padrões, eles devem ser divulgados em toda organização por meio de reuniões, comunicados, entre outros. Assim, para se evitarem possíveis confusões, devem-se estabelecer a data de início, as áreas afetadas e o local da nova sistemática, para que a aplicação do padrão ocorra de forma sincronizada em toda a organização. O treinamento para os funcionários que estão cientes e aptos a desenvolver a nova metodologia operacional padrão deve ser de preferência no local de trabalho, fornecendo recursos que são essenciais para a eficiência do treinamento.

Sendo assim, esses padrões devem ser monitorados regularmente, com o propósito de averiguar o cumprimento do padrão; fazendo desse modo, com que a empresa evite o aparecimento de um problema resolvido por causa do descumprimento dos padrões estabelecidos (ARAÚJO, 2017).

\section{CONSIDERAÇÕES FINAIS}

As ferramentas da qualidade como BPF, Procedimentos Operacionais Padronizados e Instruções de Trabalho são fundamentais para o crescimento das indústrias. Contudo a elaboração e manutenção dessas ferramentas são muito complexas e requerem o uso integrado de alguma ferramenta que auxilie nestas etapas, sendo o ciclo PDCA uma ótima indicação.

Portanto, torna-se possível afirmar que o uso do ciclo PDCA como ferramenta da qualidade usados de forma integrada, para a melhoria contínua da qualidade da empresa, por meio da elaboração de planos de ação visando aumentar o rendimento da empresa, pode contribuir notadamente na melhoria da organização. 


\section{REFERÊNCIAS}

ANDRADE, F; MELHADO, S. O método de Melhorias PDCA. Disponível em <http://publicacoes.pcc.usp.br/ PDF/BTs_Petreche/BT371-\%20Andrade.PDF>. Acesso em: 20 ago. 2019.

ARAUJO, Fernando de. APLICAÇÃO DO MÉTODO PDCA PARA SOLUÇÃO DE PROBLEMAS: ESTUDO DE CASSO EM UMA ALIMENTÍCIA NO TRIÂNGULO MINEIRO. In: ENCONTRO NACIONAL DE ENGENHARIA DE PRODUCAO, 37. 2017, Joinville. Joinville: Enegep, 2017. p. 12 - 27. Disponível em:

<http://www.abepro.org.br/biblioteca/TN_STO_239_386_31396.pdf>. Acesso em: 20 ago. 2019.

ARRUDA, José R. C. Políticas \& Indicadores de Qualidade na Educação Superior. Rio de janeiro: Qualitymark/Dunya, 1997.

Associação Brasileira das Indústrias da Alimentação (ABIA). Faturamento 2016 [internet]. Disponível em <https://www.abia.org.br/vsn/anexos/faturamento2016.pdf>. Acesso em: 19 ago. 2019.

CAMPOS, V. F.. Gerenciamento da rotina do trabalho do dia-a-dia. Belo Horizonte: Editora de Desenvolvimento Gerencial, 2001.

CAMPOS, Vicente F. Controle da Qualidade Total. $3^{\text {a }}$ ed. Rio de Janeiro: Editora Bloch, 1992.

COLTRO, Alex. A gestão da qualidade total e suas influências na competitividade empresarial. Caderno de Pesquisas em Administração, São Paulo, v. 1, n. 2, 1996.

Disponível em: . Acesso em: 28 abr. 2019.

FONSECA, Augusto V. M.; MIYAKE, Dario I. Uma análise sobre o ciclo PDCA como um método para solução de problemas da qualidade. In: ENCONTRO NACIONAL DE ENGENHARIA DE PRODUÇÃO, 26, 2006, Fortaleza. Anais do XXVI Enegep. Disponível em: . Acesso em: 28 abr. 2019.

GIL, A.C. Métodos e técnicas de pesquisa social. São Paulo: Atlas, 2007.

MELO, C. P. Caramori, E. J. PDCA Método de melhorias para empresas de Manufaturas - versão 2.0. Belo Horizonte: Fundação de Desenvolvimento Gerencial, 2001.

QUINQUIOLO, J. M. Avaliação da Eficácia de um Sistema de Gerenciamento para Melhorias Implantado na Área de Carroceria de uma Linha de Produção Automotiva. 2002. 110 f. Dissertação (Mestrado). Programa de Pós-Gradução em Administração de Empresas, Universidade de Taubaté. Taubaté, 2002. Disponível em: . Acesso em 22 abr. 2019. 


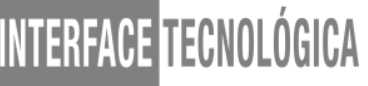

SHIBA, S.; GRAHAM, A.; WALDEN, D. TQM: Quatro Revoluções na Gestão da Qualidade. Ed. Bookman, 1997.

SLACK, Nigel. Administração da Produção. 8. ed. São Paulo: Atlas, 2018

WERMUTH, Tiago Pacheco. APLICAÇÃO DE FERRAMENTA DE QUALIDADE EM UMA INDÚSTRIA PROCESSADORA DE FRUTAS DE SANTO ANTÔNIO DA

PATRULHA - RS. 2018. 42 f. TCC (Graduação) - Curso de Engenharia Agroindustrial IndÚstrias - AlimentÍcias, Universidade Federal do Rio Grande - Furg, Santo AntÔnio da Patrulha - Rs, 2018. Disponível em:

<https://sistemas.furg.br/sistemas/sab/arquivos/conteudo_digital/48b26cdc0a66882e6a4530d4 00918902.pdf>. Acesso em: 19 ago. 2019. 